\title{
Noise Reduction Effect of Porous Asphalt Pavement Based on Acoustic-Structure Coupling Model
}

\author{
Jiakun Yang ${ }^{1 *}$, Zhou Qiu², Chuanfeng Zheng ${ }^{3}$ \\ ${ }^{1}$ School of Construction Engineering, Jilin University, Changchun 130026, China \\ ${ }^{2}$ Northeast Regional Headquarters of China Communications Construction Co., Ltd., Shenyang 11000, China \\ ${ }^{3}$ School of Transportation, Jilin University, Changchun 130026, China
}

Corresponding Author Email: 648421457@qq.com

https://doi.org/10.18280/eesrj.080206

Received: 16 March 2021

Accepted: 22 May 2021

\section{Keywords:}

traffic noise, porous asphalt pavement, porosity, pore depth, sound absorption and noise reduction, finite element

\begin{abstract}
Nowadays, the traffic noise problem is becoming increasingly prominent. In order to study the influence of porosity and pore depth on the noise reduction performance of asphalt pavement, this paper used the software COMSOL Multiphysics to establish acoustic-structure coupling models, and simulated the sound absorption and noise reduction effect of asphalt pavement under 10\%, 15\%, 20\%, 25\%, and 30\% porosity; moreover, under the condition of unchanged asphalt pavement porosity, the sound absorption and noise reduction effect of asphalt pavement under $3 \mathrm{~cm}, 4 \mathrm{~cm}, 5 \mathrm{~cm}, 6 \mathrm{~cm}$, and $7 \mathrm{~cm}$ pore depth was studied as well. The research results reveal that, with the increase of porosity and pore depth, the sound absorption and noise reduction effect of asphalt pavement gets better.
\end{abstract}

\section{INTRODUCTION}

Now people are attaching greater importance to noise pollution. There're many types of noise pollution, such as construction noise, traffic noise, and industrial noise, etc. In recent years, as urban population grows continuously, the number of vehicles is increasing, the traffic volume is increasing, and the adverse influence of traffic noise on people's living environment is becoming more serious. According to statistics, traffic noise accounted for $20.1 \%$ of the total annual environmental noise in 2001, while this number increased to more than $30 \%$ in 2004 [1]. The evergrowing transportation demands and the fast road construction have inevitably brought serious traffic noise pollution, especially in cities with large populations, traffic noise accounted for more than $75 \%$ of the total urban noise. The tire/road noise and dynamic noise generated by vehicles are the main sources of traffic noise. When the vehicle speed exceeds $50 \mathrm{~km} / \mathrm{h}$, the main source of traffic noise is the tire/road noise [2]. Therefore, to control traffic noise pollution, it is necessary to analyze the root causes of tire/road noise and develop new-type noise reduction pavement.

\section{TRAFFIC NOISE HAZARD AND TIRE/ROAD NOISE GENERATION MECHANISM}

\subsection{Traffic noise hazard}

From a physics point of view, noise is usually defined as the sound produced when the sound source vibrates irregularly. From a physiology point of view, noise interferes with the sound that people want to hear, and all sounds that hinder people's life, work, and study are called the noise. Traffic noise refers to the noise generated in the process of transportation, with the increase of vehicle number, traffic noise hazard is getting more and more serious, which is mainly reflected in the following aspects [3].

\subsubsection{Harm of traffic noise to people}

Human ears are most sensitive to sound of $1000-4000 \mathrm{~Hz}$. The tire/road noise mainly comes from vibration noise and pumping noise. Generally, the frequency of vibration noise won't exceed $1200 \mathrm{~Hz}$, and the frequency of pumping noise is usually between $1000-5000 \mathrm{~Hz}$. Studies have shown [4] that when people are exposed to high-frequency and high-decibel noise environment for a long time, it will cause serious physical and psychological damages. Physiologically, noise can cause ear discomfort, such as earache, tinnitus, hearing damage, affect sleep quality, cause abnormalities in human body circulatory system, which would even lead to cardiovascular, cerebrovascular, nervous system, and digestive system diseases; psychologically, high-frequency and high-decibel noise will cause irritability, nervousness, mood swings, neurasthenia, and inattention, etc.

\subsubsection{Harm of traffic noise to buildings}

Traffic noise will cause varying degrees of damages to surrounding buildings. The vibration of the road structure caused by the driving vehicles will spread to the surroundings through the stratums, and the nearby buildings will produce corresponding vibrations, if the natural frequency of a building is the same as the frequency of traffic noise, the building will resonate, and the structure of the building will be damaged, and the shock resistance of the structure will be weakened, in severer cases, the building might collapse.

\subsubsection{Harm of traffic noise to social economy}

Traffic noise can affect economic benefits, leading to an increase in urban operating costs. In areas that are severely 
affected by traffic noise pollution, the production and economic benefits of real estates, factories, and business districts will be reduced to varying degrees. Moreover, traffic noise also has a direct impact on the land value and house price of surrounding areas; with the improvement of people's living quality, the cost spent on noise elimination is also increasing.

\subsection{Tire/road noise generation mechanism}

Traffic noise mainly comes from the dynamic noise and tire/road noise of vehicles. The control of dynamic noise mainly depends on the technical level of vehicle manufacturing, and the control of tire/road noise mainly depends on the improvement of the design of pavement structure. When a vehicle is traveling at a low speed, the dynamic noise of the vehicle itself is the main noise source; when the vehicle is traveling at a speed greater than $50 \mathrm{~km} / \mathrm{h}$, the noise generated by the contact between the road surface and the tire is the main noise source. This paper targets at the noise generated when the tire interacts with the road surface.

\subsubsection{Air pumping effect}

When a vehicle is running, column cavities will be formed in the space enclosed by the surface texture of the tire and the road surface. In the contact process, the tire will generate compressive deformation in the lateral, vertical, and radial directions. The compression of the space of tire surface texture will squeeze the air in the cavities and explode it into the atmosphere. At the same time, the air contained in the pavement pores will also be squeezed out by the action of the tire, then, when the tire leaves the pores squeezed by the air, the air will refill the cavities of the tire surface texture and the pavement pores that are previously squeezed, and this process would form the air-pumping effect, see Figure 1. As the tire rotates, the air pumping effect occurs periodically, causing the air to form a sparse-dense wave; this process will cause the air to vibrate violently, forming a unipolar sub-noise source, the generated noise has a high frequency, which is also called the air pressure noise between the tire and the road surface [5]. Zhang and Huang [6] have pointed out in their study that, in tire/road noise, the impact of air pressure noise is the greatest.

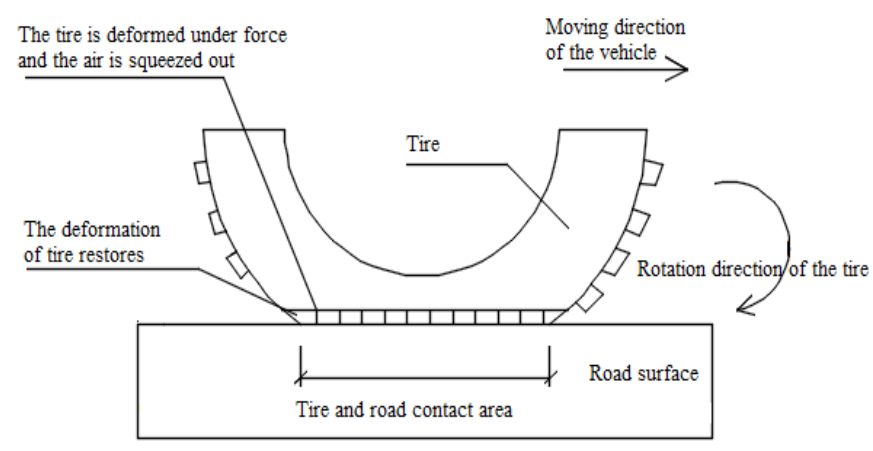

Figure 1. Aire pressure noise

\subsubsection{Vibration noise}

Vibration noise is mainly caused by the vibration of tires when rolling on roads with different surface structures. It mainly includes the following three aspects [7]: first, the elastic vibration noise, when the rubber blocks on the tire surface impact the road surface, the tire will deform, and the tire sidewall and tire tread will produce vibration noise, the impact of the texture blocks can be compared to small rubber hammers hitting on the road surface, see Figure 2, and this is the main source of the tire/road vibration noise. Second, the self-excited vibration noise, when the vehicle starts, or turn or brake suddenly, the tread will produce local self-excited vibration relative to the road surface, and the vibration will be transmitted to the body of the vehicle through the suspension device, and the self-excited vibration noise is the indirect vibration noise produced by the vehicle body. Third, the road vibration noise, which is the noise generated by the vibration of road surface under the action of tires when the vehicle is running on it.

$\stackrel{\text { Moving direction of the vehicle }}{\longrightarrow}$

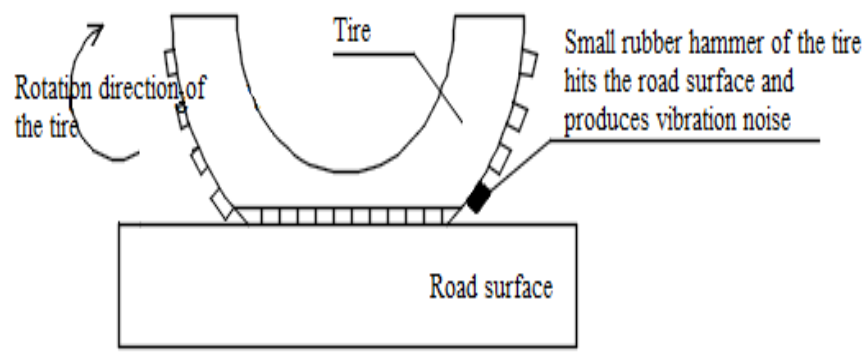

Figure 2. Vibration caused by the impact of tire texture/road surface

\section{THE NOISE REDUCTION PRINCIPLE OF POROUS ASPHALT PAVEMENT}

Since pores of different types will be formed in the porous asphalt pavement, the pores are interconnected, and the pores inside the pavement connect to the atmosphere through the road surface pores, this feature can prevent the compressed high-speed airflow from being directly discharged into the outside atmosphere, the compressed air can smoothly enter the road surface pores and diffuse to the surroundings at a relatively large speed, thereby reducing the air pumping effect between the road surface and the tire, as shown in Figure 3(a). In addition, when the sound waves touch the sound-absorbing structure of the road surface, some sound waves will be directly reflected to the atmosphere by the road surface, and the remaining sound waves will enter the pores inside the sound-absorbing structure of the road surface, causing the air in the pores to vibrate, then the vibrating air rubs against the inner wall of the pores, according to the heat conduction effect, kinetic energy of air vibration is converted into heat energy, which will then be dissipated, in such repeated process, the energy of the sound waves decreases gradually, the Helmholtz resonator shown in Figure 3(b) can reflect the sound absorption and noise reduction mechanism of the porous asphalt pavement. When the frequency of the sound wave injected into the pavement is consistent with the natural vibration frequency of the resonator, resonance will occur, at this time, the speed of air reciprocating in the cavity reaches the maximum, the energy loss of friction also reaches the maximum, and the pavement structure will absorb the sound energy of the incident sound waves to the greatest extent. 


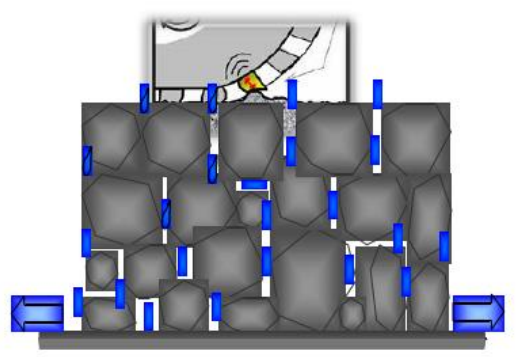

(a) Air flow in pores [8]

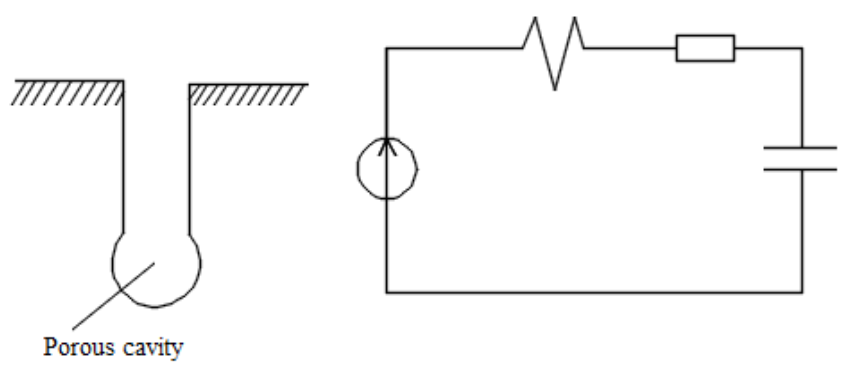

(b) Helmholtz resonator

Figure 3. Noise reduction principle of porous asphalt pavement

\section{FINITE ELEMENT MODEL OF THE SOUND ABSORPTION STRUCTURE OF POROUS ASPHALT PAVEMENT}

In this study, the software COMSOL Multiphysics was employed to establish the acoustic-structure coupling model. The interaction between the sound-absorbing structure of the porous asphalt pavement and the sound waves of the flow field is a two-way coupling problem. The "Pressure AcousticsTransient" interface in COMSOL is mainly used to calculate the pressure changes when sound waves propagate in fluids under static simulation conditions, it could be applied to the transient simulation of any transient fields and sources and solving scalar wave equations in the time domain, it can offer models for studies concerning transient modes and modal order reduction, and solve equations in the frequency domain based on available boundary conditions, therefore, the "Pressure Acoustics-Transient" and the "Solid Mechanics" physical fields were added in the three-dimensional space for simulation.

The asphalt pavement contains various types of pores, including the connected type, the semi-closed type, and the fully-closed type; among these types of pores, only the connected and semi-closed pores can contribute to sound absorption and noise reduction, therefore pores of these two types are called the effective pores. Since finite elements can hardly separate different types of pores to reflect the real pavement conditions, in order to accurately control the variables and objectively reflect the impact of the changes in porosity and pore size on the sound absorption and noise reduction performance of asphalt pavement, in this paper, the pores were all set to be rectangular-shaped effective pores.

The sound-absorbing structure of the pavement was set to be a cuboid with a size of $3 \mathrm{~cm} \times 3 \mathrm{~cm} \times 8 \mathrm{~cm}, 5 \times 5=25$ pores were uniformly distributed, the initial depth of the pores was $\mathrm{h}=7 \mathrm{~cm}$, pore diameter was a, pore distance was $\mathrm{c}, \mathrm{a}+\mathrm{c}=0.6 \mathrm{~cm}$; the control of the porosity of the sound-absorbing structure of the asphalt pavement was realized by adjusting the pore diameter and pore distance, and the corresponding relationship is shown in Table 1. The sound-absorbing structure of the asphalt pavement established in COMSOL is shown in Figure 4.

Table 1. Pore diameter, distance, and depth corresponding to each porosity value

\begin{tabular}{cccc}
\hline Porosity & $\begin{array}{c}\text { Pore diameter } \\
\mathrm{a} / \mathrm{cm}\end{array}$ & $\begin{array}{c}\text { Pore distance } \\
\mathrm{e} / \mathrm{cm}\end{array}$ & $\begin{array}{c}\text { Pore depth } \\
\mathrm{h} / \mathrm{cm}\end{array}$ \\
\hline $10 \%$ & 0.203 & 0.397 & 7 \\
$15 \%$ & 0.257 & 0.343 & 7 \\
$20 \%$ & 0.280 & 0.320 & 7 \\
$25 \%$ & 0.320 & 0.280 & 7 \\
$30 \%$ & 0.351 & 0.249 & 7 \\
\hline
\end{tabular}

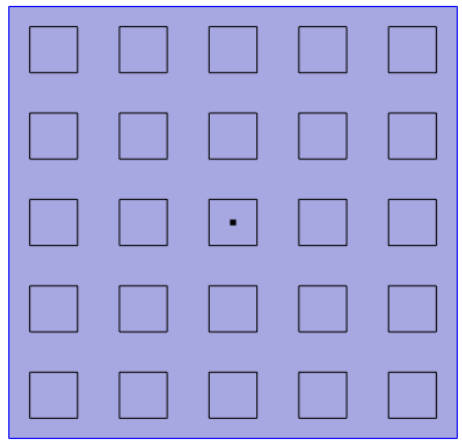

(a) Pore distribution of the sound-absorbing structure of the pavement

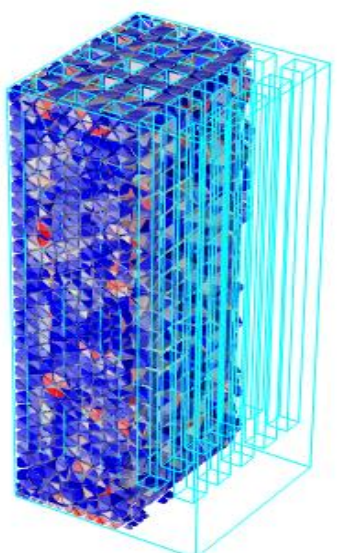

(b) The sound-absorbing structure of the pavement in 3D

Figure 4. Sound-absorbing structure of porous asphalt pavement

A sound source point A was set directly above the soundabsorbing structure of the porous asphalt pavement, from point A, a series of simple harmonic sound waves with a certain frequency and amplitude was released; then in subsequent finite element analysis, the noise reduction values were calculated based on the obtained incident sound pressure and reflected sound pressure at the sound source point, then, the sound absorption and noise reduction performance of the sound-absorbing structure of the pavement under different states could be obtained. In order to clearly distinguish the incident sound wave from the reflected sound wave and obtain steady-state solution, the distance between the reflection surface of the sound-absorbing structure of the asphalt 
pavement and the sound source point must be greater than at least 6 wavelengths [9], therefore, in the proposed model, point A was set $60 \mathrm{~cm}$ directly above the reflection surface of the sound-absorbing structure of the asphalt pavement. The material properties in each phase were given under the conditions of an atmospheric pressure of $1 \mathrm{~atm}$ and a temperature of $293.15 \mathrm{~K}$. In the air phase, in the asphalt mixture phase, the density of the asphalt mixture was 2450 $\mathrm{kg} / \mathrm{m}^{3}$, the elastic modulus was $1300 \mathrm{MPa}$, and the Poisson's ratio was $0.2 \mathrm{nu}$.

In the transient pressure acoustics module, the fluid model was linear elastic, the type of periodicity was consecutive, the type of sound wave was plane wave radiation. In the solid mechanics module, the sound-absorbing structure model was linear elastic material, and the type of periodicity was consecutive. Since the selected sound-absorbing structure of the asphalt pavement was symmetrical, in order to simplify the calculation process, one of the sound-absorbing structure cavities could be selected to perform the calculation; to obtain more accurate calculation results, the maximum cell size of the meshes was controlled to be $340 / f / 12 m$, wherein $\mathrm{f}$ was the frequency of the harmonic sound wave.

\section{FINITE ELEMENT ANALYSIS OF THE INFLUENCE OF POROSITY ON THE NOISE REDUCTION EFFECT OF ASPHALT PAVEMENT}

In order to study the influence of porosity on the noise reduction performance of the pavement, the porosity of the sound-absorbing structure was set to be $10 \%, 15 \%, 20 \%, 25 \%$, and $30 \%$, respectively. Since the air-pumping noise frequency of the porous asphalt pavement was mainly concentrated between $900 \mathrm{~Hz}-1500 \mathrm{~Hz}$, the vibration noise frequency was mainly concentrated between $300 \mathrm{~Hz}-700 \mathrm{~Hz}$, and the airpumping noise was the main source of noise, therefore, the frequency of the simple harmonic sound wave applied in COMSOL had been set to $900 \mathrm{~Hz}$, and the amplitude was set to $1 \mathrm{~Pa}$. Through the acoustic-structure boundary, the "pressure acoustics-transient" and the "solid mechanics" physical fields were coupled, then, by clicking the "research" step in COMSOL and performing calculations, the change of the total sound pressure field over time at sound source point A could be obtained. Figure 5 shows the change of the total sound pressure field over time at sound source point $A$ under different porosity values of the porous asphalt pavement.

As shown in Figure 5, according to the change of sound pressure at sound source point A, after processed by the soundabsorbing structure of the pavement, the sound pressure value of the sound wave was smaller than the initial incident sound pressure. In order to reflect the sound absorption and noise reduction effect of the sound-absorbing structure more intuitively, the sound pressure was converted into sound pressure level using the following formula. The sound pressure level is the indicator used to define the sound size in the acoustic field, its unit is $\mathrm{dB}$.

$$
L_{P}(d B)=20 \lg \frac{p}{p_{r e}}\left(p_{r e}=2 \times 10^{-5} N / m^{2}\right)
$$

where, $L_{p}$ is the sound pressure level; $P$ is the measured sound pressure; $P_{r e}$ is the reference sound pressure. The sound pressure levels corresponding to the incident sound pressure and the reflected sound pressure can be calculated by the above formula, by finding the difference between the two, the noise reduction value after the sound waves were processed by the sound-absorbing structure of the pavement could be obtained, and the change of noise reduction value with porosity is shown in Figure 6.

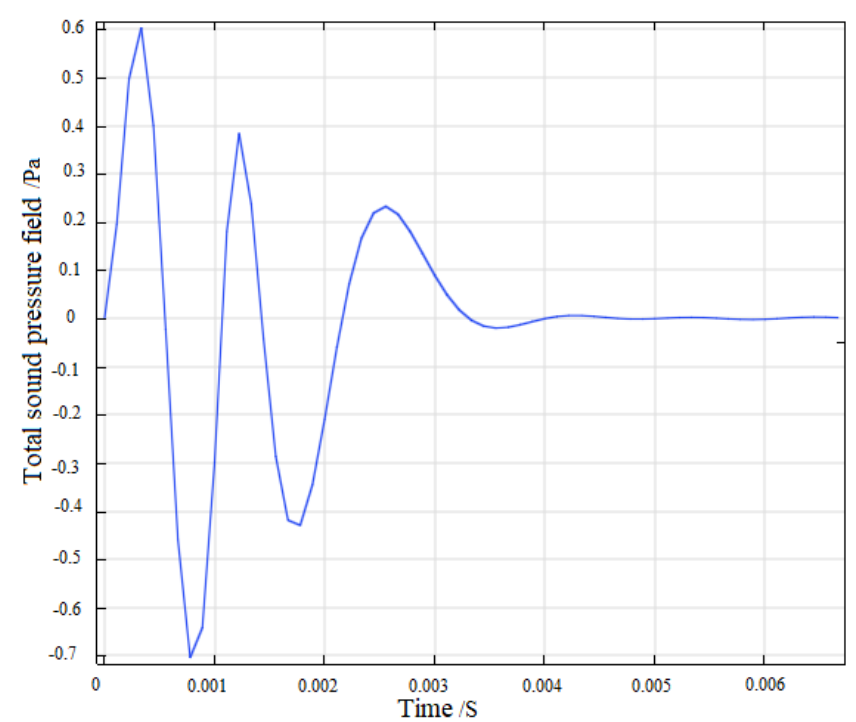

(a) Change of sound pressure over time under $10 \%$ porosity

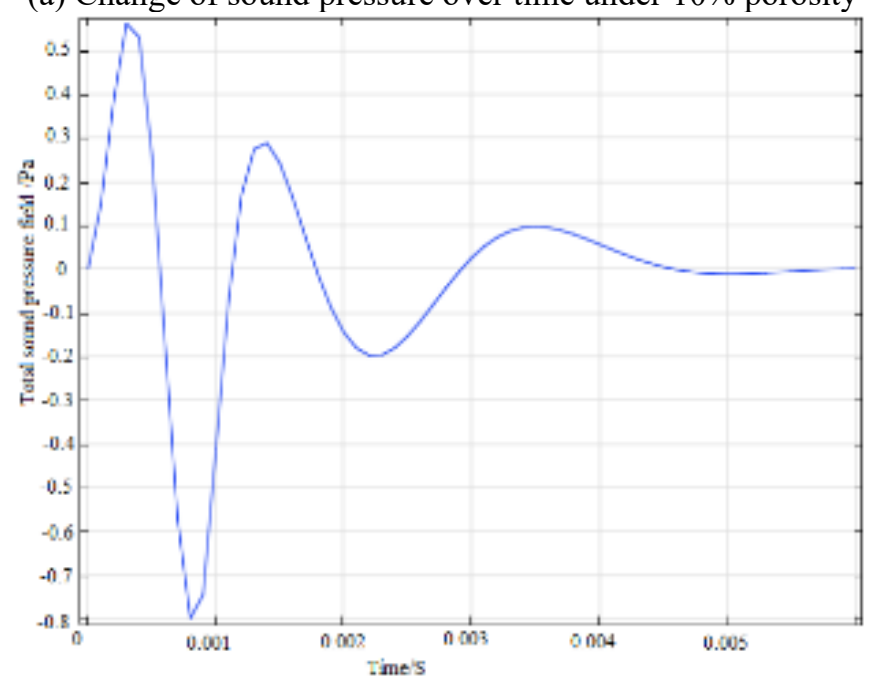

(b) Change of sound pressure over time under $15 \%$ porosity

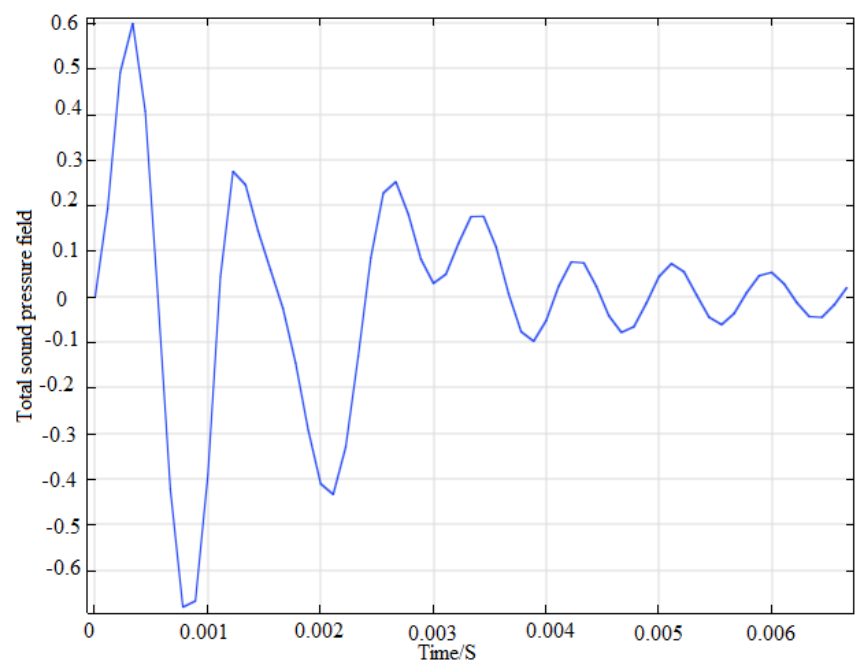

(c) Change of sound pressure over time under $20 \%$ porosity 


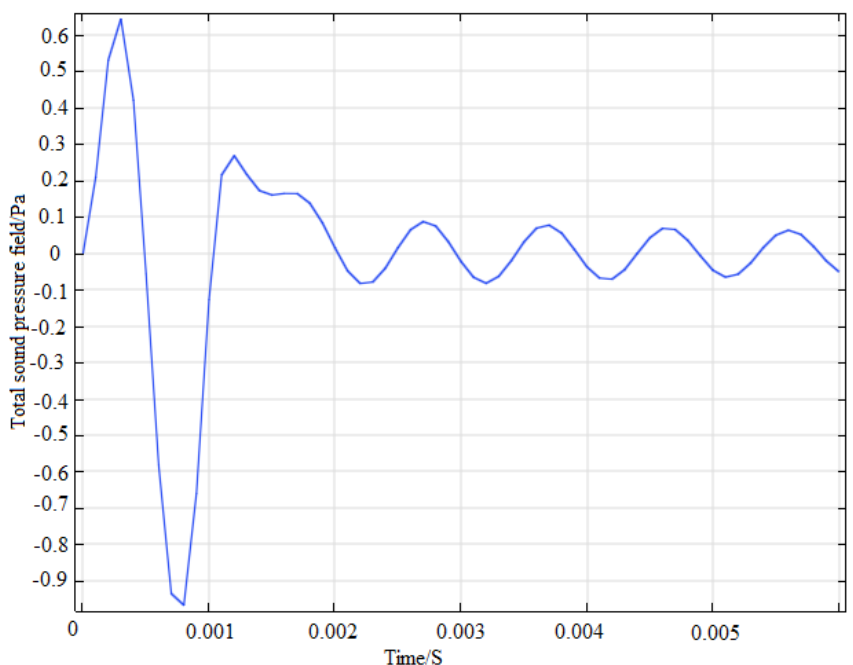

(d) Change of sound pressure over time under $25 \%$ porosity

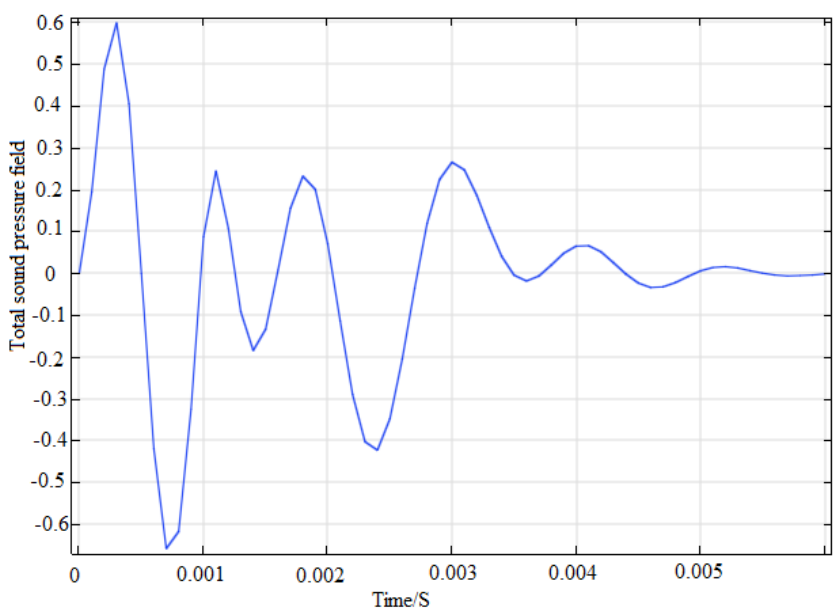

(e) Change of sound pressure over time under $30 \%$ porosity

Figure 5. Change of sound pressure at sound source point $A$ under different porosity values

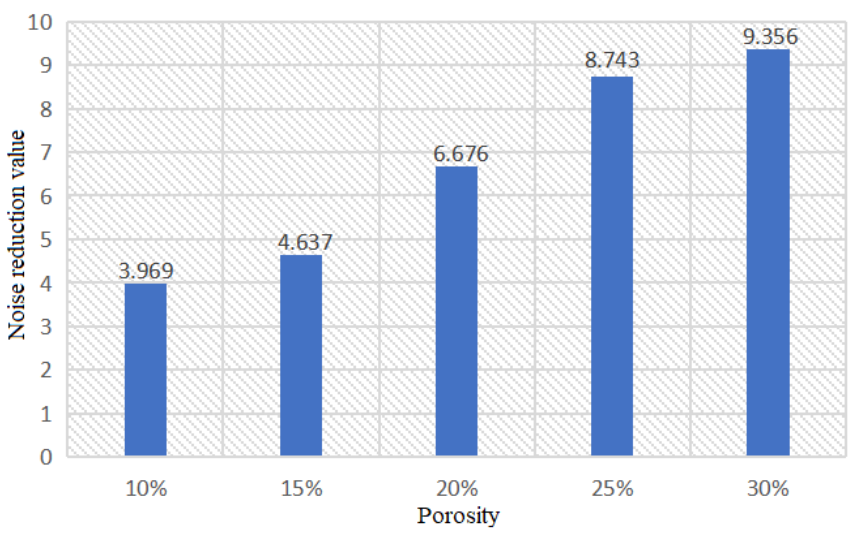

Figure 6. Noise reduction corresponding to each porosity value

Result analysis: as shown in Figure 6, with the increase of porosity, the noise reduction value becomes larger; when porosity changes from $10 \%$ to $15 \%$, the noise reduction value increases a bit but the increment is small; when the porosity changes from $15 \%$ to $20 \%$ and from $20 \%$ to $25 \%$, the increment of noise reduction value is more obvious; when the porosity changes from $25 \%$ to $30 \%$, the noise reduction value tends to be stable. In terms of road performance, porosity increase would result in a decrease in the maximum flexuraltensile strain, and the low-temperature crack resistance of the pavement would become worse, this is due to the low asphalt content in the asphalt mixture with large porosity. Therefore, it's determined that $25 \%$ is the best porosity, and this result is also consistent with the research conclusion of Wang Tao's experiments [10]

\section{FINITE ELEMENT ANALYSIS OF THE INFLUENCE OF PORE SIZE ON NOISE REDUCTION EFFECT}

In actual road pavement, there're pores of different shapes, sizes, and depths, and for these different types of pores, their sound absorption and noise reduction effects are different as well. According to a research report of Clemson University in the United States [11], under the condition of a same pavement porosity, with the decrease of pore size, the effective surface area of the pores increases. When sound enters the pores and propagates, the energy loss of sound waves after entering pores with smaller size is greater than that after entering pores with larger size, then, we can see that the sound absorption and noise reduction performance of pavement with smaller pores is better than that with larger pores. In this section, this paper studied the influence of pore depth on the noise reduction performance of the sound-absorbing structure of the pavement under the condition of same porosity. In COMSOL, the pore depth $h$ value in the initial pavement sound-absorbing structure layer model corresponding to each porosity was adjusted from $7 \mathrm{~cm}$ to $3 \mathrm{~cm}, 4 \mathrm{~cm}, 5 \mathrm{~cm}$, and $6 \mathrm{~cm}$ respectively, then, the simulations were performed one by one to calculate the noise reduction values. With pavement with $10 \%$ porosity as an example, under different pore depth $\mathrm{h}$ values, the corresponding pore diameter a values and pore distance $\mathrm{c}$ values are listed in Table 2.

Table 2. Corresponding relations among pore depth, pore diameter, and pore distance under a porosity of $10 \%$

\begin{tabular}{ccc}
\hline Pore depth $\mathrm{h} / \mathrm{cm}$ & Pore diameter $\mathrm{a} / \mathrm{cm}$ & Pore distance $\mathrm{c} / \mathrm{cm}$ \\
\hline 3 & 0.309 & 0.191 \\
4 & 0.268 & 0.332 \\
5 & 0.240 & 0.360 \\
6 & 0.219 & 0.381 \\
7 & 0.203 & 0.397 \\
\hline
\end{tabular}

Table 3. Change of noise reduction value with pore depth under each porosity

\begin{tabular}{cccccc}
\hline \multirow{2}{*}{ Porosity } & 3 & \multicolumn{5}{c}{ Pore depth h/cm } \\
\hline $10 \%$ & $2.169 \mathrm{~dB}$ & $2.487 \mathrm{~dB}$ & $2.609 \mathrm{~dB}$ & $3.515 \mathrm{~dB}$ & $3.969 \mathrm{~dB}$ \\
$15 \%$ & $2.537 \mathrm{~dB}$ & $3.245 \mathrm{~dB}$ & $3.109 \mathrm{~dB}$ & $4.306 \mathrm{~dB}$ & $4.637 \mathrm{~dB}$ \\
$20 \%$ & $3.277 \mathrm{~dB}$ & $3.986 \mathrm{~dB}$ & $4.348 \mathrm{~dB}$ & $5.959 \mathrm{~dB}$ & $6.676 \mathrm{~dB}$ \\
$25 \%$ & $3.943 \mathrm{~dB}$ & $5.331 \mathrm{~dB}$ & $6.254 \mathrm{~dB}$ & $6.772 \mathrm{~dB}$ & $8.743 \mathrm{~dB}$ \\
$30 \%$ & $4.379 \mathrm{~dB}$ & $6.048 \mathrm{~dB}$ & $6.643 \mathrm{~dB}$ & $7.435 \mathrm{~dB}$ & $9.356 \mathrm{~dB}$ \\
\hline
\end{tabular}

The calculation results are shown in Table 3, Figure 7 gives the change of noise reduction value with pore depth under each porosity.

Result analysis: according to Figure 7, under each porosity, with the increase of pore depth, the noise reduction value of the sound-absorbing structure of the pavement increases as well, and this calculation result is consistent with the research report of Clemson University [11]. When sound propagates in 
the pores, the energy loss of sound waves after entering pores with smaller size is greater than that after entering pores with larger size, the noise reduction performance of the pavement with smaller pores is better than that of pavement with larger pores. According to Figure 7, when porosity changes between $15 \%-20 \%$ and $20 \%-25 \%$, the increment of noise reduction value is more obvious; when porosity changes between $25 \%$ $30 \%$, the noise reduction value tends to be stable.

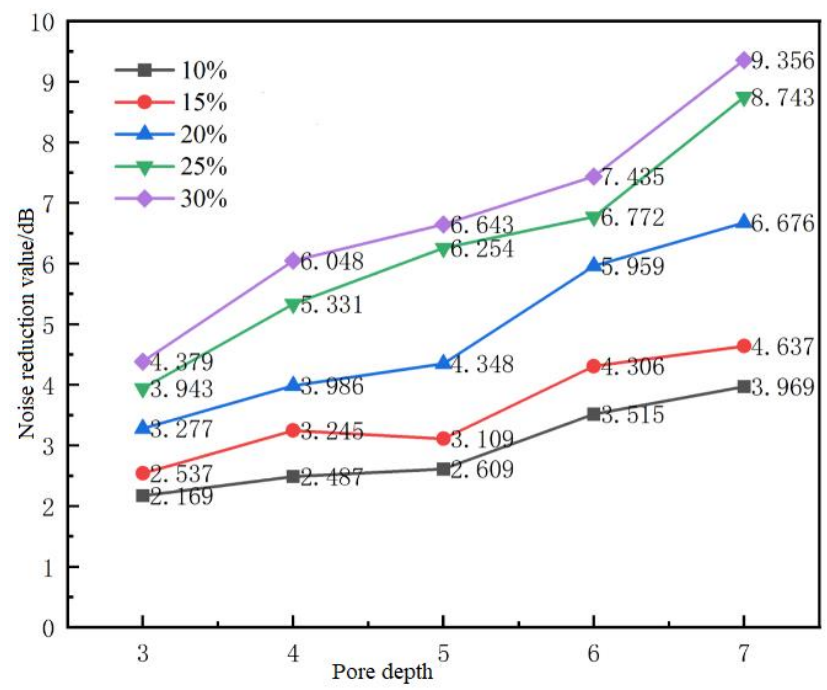

Figure 7. Change of noise reduction value with pore depth under each porosity

\section{CONCLUSIONS}

This paper discussed the hazards and generation mechanism of traffic noise, and used the finite element method to simulate the sound absorption and noise reduction performance of porous asphalt pavement in software COMSOL Multiphysics. The influence of porosity and the pore depth on the sound absorption and noise reduction performance of the asphalt pavement was explored, and the following conclusions were drawn:

(1) With the increase of porosity, the noise reduction value becomes larger, when the porosity is $10 \%$, the maximal noise reduction value is $3.969 \mathrm{~dB}$; when the porosity is $15 \%$, this value is $4.637 \mathrm{~dB}$; when the porosity is $20 \%$, this value is $6.676 \mathrm{~dB}$; when the porosity is $25 \%$, this value is $8.743 \mathrm{~dB}$; when the porosity is $30 \%$, this value is $9.356 \mathrm{~dB}$. Combining with the road performance, it can be determined that the best porosity is $25 \%$.

(2) Under a same porosity value, as the pore depth increases, the noise reduction value increases as well, the energy loss of sound waves when propagating in pores with smaller size is greater than that propagating in pores with larger size; under the premise of constant porosity, the greater the pore depth, the better the sound absorption and noise reduction performance of the pavement.

\section{REFERENCES}

[1] Yang, Z.Y. (2007). Noise accompanied by cars rushing into urban life. Scientific Life, 4: 39.

[2] Iwao, K., Yamazaki, I. (1996). A study on the mechanism of tire/road noise. Jsae Review, 17(2): 139144. https://doi.org/10.1016/0389-4304(95)00004-6

[3] Liu, H.L. (2007). Environmental Noise Control. Heilongjiang: Harbin Institute of Technology Press.

[4] Xie, Y. (2017). External noise characteristics and spectrum evaluation of asphalt pavement under vehicletire-road coupling. Southeast University. https://doi.org/CNKI:CDMD:2.1018.128608

[5] Wei, J.J., Kong, Y.J. (2004). Research on the noise reduction mechanism of multi-porous low noise asphalt pavement. Journal of Hei long jiang Institute of Technology, 18(1): 11-13, 19. https://doi.org/10.3969/j.issn.1671-4679.2004.01.004

[6] Zhang, L.H., Huang, X.M. (2004). Finite element analysis of tire/road noise. Chinese Journal of Environmental Engineering, 2(12): 1695-1697.

[7] Hou, Z.Y., Pang, M.B. (2004). Acoustic analysis of low noise asphalt concrete pavement. Highway, 6: 22-25. https://doi.org/10.3969/j.issn.0451-0712.2004.06.006

[8] Yu, W.T. (2016). Research on noise reduction effect of low-noise asphalt pavement reconstruction. Chongqing Jiaotong University.

[9] Wang, H.C., Huang, X.M., Liao, G.Y., Xue, G.Q. (2011). Finite element analysis of sound absorption and noise reduction of double-layer porous asphalt pavement. Techniques and Equipment for Environmental Pollution Control, 5(12): 2910-2914.

[10] Wang T. (2016). Analysis of noise reduction principles and influencing factors of low noise asphalt pavement. Shandong Transportation Science and Technology, 4: 113-115, 117. https://doi.org/10.3969/j.issn.16738942.2016.04.037

[11] Shatanawi, K. (2008). The effects of crumb rubber particles on highway noise reduction-A laboratory study. 